Article

\title{
The Characterization of Biological Organization, Abstraction, and Novelty in Biomimetic Design
}

\author{
Devesh Bhasin * and Daniel A. McAdams \\ Department of Mechanical Engineering, Texas A\&M University, College Station, TX 77840, USA; \\ dmcadams@tamu.edu \\ * Correspondence: devesh1904@tamu.edu; Tel.: +1-979-739-7752
}

Received: 2 October 2018; Accepted: 7 December 2018; Published: 11 December 2018

check for updates

\begin{abstract}
Through billions of years of evolution, a latent record of successful and failed design practices has developed in nature. The endeavors to exploit this record have resulted in numerous successful products in various fields of engineering, including, but not limited to, networking, propulsion, surface engineering, and robotics. In this work, a study of existing biomimetic designs has been carried out by categorizing the designs according to the biological organizational level, the abstraction level, and a novelty measure. The criterion of novelty has been used as a partial measure of the quality of bio-inspired and biomimetic designs already introduced, or ready to be introduced to the market. Through this review and categorization, we recognize patterns in existing biomimetic and bio-inspired products by analyzing their cross-categorical distribution. Using the distribution, we identify the categories which yield novel bio-inspired designs. We also examine the distribution to identify less explored areas of bio-inspired design. Additionally, this study is a step forward in aiding the practitioners of biomimetics in identifying the categories which yield the highest novelty products in their area of interest.
\end{abstract}

Keywords: biomimetics categorization; biomimetics distribution; patterns in biomimetics; novel bio-inspired design

\section{Introduction}

In recent years, inspiration from biology has noticeably influenced engineering design. There are numerous successful biological entities which inspire solutions to human challenges and stimulate the creation of new engineering knowledge [1-4]. Biology has the potential to provide tools and processes to develop solutions that are far more resilient and sustainable than their man-made counter-parts $[4,5]$. This is because biology solves problems largely by manipulating the factors ignored by technology [4]. The process of biomimetics is based on cross-domain analogies and requires expertise in two fundamentally disparate domains of engineering and biology [6,7]. Humans have used biology as an inspiration to solve engineering problems for almost 3000 years [4]. Yet, potential users still face challenges within a bioinspired or biomimetic design process, resulting in fragmented and isolated research areas [2,4]. This is due to the reason that biological problem-solving strategies generally cannot be directly carried over from biology to technology but rather require a creative implementation method $[4,8]$.

Typically, designers incorporating the use of biomimetics in their design strategies face challenges during the search and selection of inspiration from the large biological databases, the scaling of biological strategies to the problem at hand, and generating nonconflicting biomimetic solutions for integrated engineering design [9].

In order to clearly identify challenges faced by practitioners of bio-inspired design, Helms conducted an experiment on an undergraduate class consisting of inter-disciplinary students [6]. 
The experiment involved the conceptualization of a bio-inspired solution for a given problem. Based on the results of Helms, eight types of errors that could occur in a biologically inspired process were identified as follows:

1. Vaguely defined problem;

2. Poor problem-solution pairing;

3. Over-simplification of complex functions;

4. Using 'off the shelf' biological solutions;

5. Simplification of optimization problem;

6. Solution Fixation;

7. Misapplied analogy;

8. Improper analogical transfer.

Shu further related the identified errors to the level of similarity between the biological phenomenon and their corresponding biomimetic concepts developed in technology [10]. Based on strategic accuracy and abstraction level of biological entities, Shu observed four similarity types: literal implementation, biological transfer, anomaly, and analogy [10,11]. Shu concluded that out of the four categories, only analogical similarity accurately transfers the strategy of a biological entity to the concept without transferring the biological entity itself [10]. Further, Waneik identified eleven underlying challenges in a four-step normative biomimetic process flow from idea to the market [3]. A compilation of 50-year progress in bio-inspired design indicated that the field of biomimetics lacks a clear method to carry-out the process [12]. The successful implementation of biomimetics in technology most critically depends upon establishing the biology-technology analogy at the appropriate level of abstraction $[10,13,14]$. This requires multi-level abstraction and suitable modification of biological strategies, which need to be made understandable for potential designers having little to no expertise in biology $[7,8]$.

To address the problems existing in biomimetic processes, efforts have been made to develop normative biomimetic processes and identify approaches that can aid the non-experts in biology and lead to a successful implementation of biomimetic strategy. Two general approaches to biomimetics have been proposed in the literature: a problem-driven approach and a solution-driven approach. What we will term as the problem-driven approach has been represented as a problem-driven approach [2,3,6,15], a problem-based approach [9,12], a challenge-based approach [12], biomimetics by analogy [16], challenge to biology [17], a top-down approach [7,8,18], and a mechanism-driven approach [19]. Although different representations exist, the high-level sequence of activities that lead to a design solution remains the same. Similarly, the solution-driven approach has been represented as a solution-driven approach [6,15], a solution-based approach [2,3,9,12], biomimetics by induction [16], biology to design [17], a bottom-up approach [7,8,18], and an organism-driven approach [19]).

The problem-driven approach largely emerges from engineers and designers seeking to utilize biological principles and strategies in both solving general design problems and innovating through limitations existing in technology. On the contrary, the solution-driven approach largely emerges from biological and life-science experts seeking to utilize their expertise in developing novel applications in technology. However, both approaches allow a systematic consideration of biological systems during solution creation or extraction phases of the design process [20]. Moreover, both processes show a similar trend in the transfer of information from the biological to technological domain and vice-versa [9]. Multiple studies have analyzed the use of the two approaches in developing biomimetic products. Generally, the motive to adopt biomimetic designs in technology is to generate multi-functional designs [21]. This motive is achieved by both problem-driven and solution-driven approaches [6]. The Bio-M database reflects that the use of solution-driven approach is more prominent among the practitioners of biomimetics [12].

The two approaches to biomimetics, in practice, do not span all the approaches followed by the practitioners of biomimetics [12,22]. Moreover, the two approaches only provide a general procedure 
to apply biomimetics in engineering design. As a method, both may fail to aid practitioners of biomimetics in identifying biological domains relevant to their area of interest.

As a static analysis, the method of categorization has the potential to illuminate that state of the art of biomimetic practice. Additionally, the review and categorization of existing biomimetic products enables the study of cases of biomimetic design beyond just the problem-driven and solution-driven approach. Such knowledge informs the design practitioner as well as the biomimetic design researcher. Through organization and categorization of biomimetic designs, different biomimetic design methods can be identified and codified. The methods of categorical distribution of bio-inspired products have been used in multiple studies to analyze the focus and trend of the practice of biomimetics [11,23,24]. The cross-categorization of biomimetic products has been analyzed to reveal prominent areas of bio-inspired design practice, and to identify the unexplored categories for bio-inspired design research [24]. The study was performed by categorizing biomimetic products based on the level of biological organization, the class of biological entity, the type of technical system developed, and the frequency of application. The analysis concluded that most existing biomimetic products are inspired by animals, and most bio-inspired technological developments take place in the area of 'Biomechatronic and Robotics'. In addition, the study identified 'Plant Kingdom' and biological organizational levels of 'molecules', 'cell', and 'population' as the less frequently explored areas, indicating future bio-inspired design research directions [24].

However, the biomimetic studies in existing literature do not consider the qualitative effect of bio-inspirations on technology, that is, novelty. While several studies have identified the significance of novelty in developing quality concepts for engineering problems, the notion of novelty in biomimetics has been emphasized by relatively fewer studies. The suggested method utilizes a qualitative metric for novelty in determining bio-inspirations for engineering designs. Additionally, the method mitigates the exhaustive search currently required to identify relevant bio-inspirations for solving engineering problems by identifying categories that lead to comparatively more novel bio-inspired designs than other categories.

In the remainder of this paper, we create and apply a categorization framework for biomimetic designs. We attempt to categorize biomimetic products based on their biological organizational level, their abstraction level and their novelty. One element of our organizational structure is a measure of the novelty of the resultant biomimetic design. Measures of design novelty are an evolving construct in design research and thus using design novelty as a categorization metric is challenging [25-27]. Nevertheless, innovation and novelty are key drivers of biomimetic design interest. We must address some notion of design innovation as we push forward the progress of biomimetic design. Our categorization framework allows a designer to review biomimetic design cases that are novel. And, thus, through following in the footsteps of previous biomimetic designers, pursue a path of exploration that has provided novel results in the past.

\section{Materials and Methods}

To analyze the state-of-the-art patterns of biomimetic design practices existing in technology, we created a sample set indicating the current practice of biomimetic design by identifying biomimetic designs available in the literature. Our goal here is to determine the categories which contribute significantly to the development of quality biomimetic products. The distinction between the definitions 'biomimetics' and 'bio-inspired' is acknowledged by the authors [28]. Biomimetics has been defined as a subset of bio-inspired design in the literature [2,28]. Although bio-inspired design and biomimetics have different effects on the product design, the final outcome of the two approaches is the same, that is, the invention of a product that has been made possible with the knowledge originating from nature [3]. In this study, we consider the final outcome of the approach, independent of the approach used to develop the product. Therefore, we find it justified to include both biomimetic and bio-inspired designs in this search and review. Subsequently, the material in the study has been labeled as 'biomimetic designs' but contains bio-inspired designs as well. 
In 2008, Vincent analyzed the trend of biomimetic products by categorizing them according to the engineering domains of application [23]. As many biomimetic designs do not end up as feasible products, Vincent proposed using biomimetic designs that had emerged or were ready to emerge as market products as the sample [23]. We used the same methodology in this research for defining the sample set for analysis. Our indicative sample set consisted of biomimetic designs that were in the implementation stage, that is, a prototype of the design existed, and/or was available to compare its performance with its respective technological counterpart, if any. Biomimetic designs that had already been implemented, for example, in commercially available products and patents were also included in the set. To avoid replication of biomimetic products within the sample set, we used the AskNature database to search for biomimetics designs [29]. The database provides a list consisting of two hundred one $(n=201)$ biomimetic design ideas. We identified the biomimetic products at or beyond implementation phase in database. The products were selected independent of the design approach being problem-driven or solution-based. We also considered the instances of biomimetic design where the abiotic phenomenon, like the water cycle, inspired the biomimetic product. The resultant sample set was further refined by using additional criteria which is described as follows:

- We only considered the designs that resulted in a tangible product/prototype. Cases of bio-inspired designs that resulted in intangible products, for example, bio-inspired optimization algorithms, were not considered as a part of the sample set. Such selection criteria helped focus our search and review without significantly affecting the scope of the study.

- Certain designs were observed which involved inspiration from multiple biological instances. In such cases, each inspiration was accounted as a separate entity in the compiled sample set. For example, bio-inspired anti-ice coatings were found to be inspired by two biological entities, lotus leaves and poison dart frog skin [30]. Therefore, the combined design was counted as two individual bio-inspired designs.

We found one hundred eighty-eight $(n=188)$ cases of biomimetic designs which satisfied the selection criteria. This sample set is non-exhaustive but serves as an indicator of the practice of biomimetic design.

Once the indicative sample set was generated, each design in the sample was categorized according to three variables: biological organizational level, abstraction level, and novelty. This categorization provides a concise way to understand important elements of the biomimetic process outcome for each design. The biological organizational level provides an indication of where the biological phenomenon was found. The abstraction level indicates the degree of similarity between the biological phenomena and its corresponding solution realized in technology. The novelty measure provides insight about the quality of the biomimetic product and its application. The categories have been discussed in detail in the subsequent sections. Further, the detailed analysis and classification of six biomimetic products has been shown as an example.

\subsection{Biological Organizational Level}

Researchers and scientists study biology by classifying biological phenomena into several levels of organization. The biological organization scale is a hierarchy of levels, ranging from molecule to biosphere. Each level is made of components of the smaller level and itself is a component of the larger level. In the context of this work, the biological organizational level indicates the level at which of the biological phenomenon serving as the inspiration to the design was identified. In the field of biomimetics, the biological organizational scale has been used to generalize biological phenomenon in order to effectively identify biological analogies for engineering design [24,31,32]. The scale originally consisted of eleven levels. However, during categorization, we observed certain patterns which led to reduction of the scale to nine levels. Most of the bio-inspirations taken from the community level were based on interactions between the populations and did not result in tangible designs. Moreover, 
other designs inspired by the phenomenon at the community level could be seen in at least one of the smaller hierarchical levels. For example, the design of shading structures was inspired by the phenomenon of shading shown by forest canopy, that is a community of trees in a forest. The same phenomenon was also found to be performed by a population of trees, hence, the smallest biological level of observation was population and not community. The biosphere level showed a similar trend. The biosphere level includes the sum of all ecosystems present on earth. Essentially, each ecosystem is capable of independent existence and are mostly isolated from each other, the phenomenon occurring in the biosphere level could be seen in at least one of the existing ecosystems. Therefore, we found it justified to omit the levels of community and biosphere from the biological organizational scale. This resulted in a final scale consisted of nine levels as following:

1. Molecule;

2. Organelle;

3. Cell;

4. Tissue;

5. Organ;

6. Organ System;

7. Organism;

8. Population;

9. Ecosystem.

In some cases, biomimetic designs were found to be inspired by biological phenomenon occurring at multiple biological levels [33]. In such cases, we selected the smallest biological level at which the phenomenon was observed. For example, self-assembly is common throughout nature and occurs at multiple scales [34]. The smallest level of its occurrence is the molecule level; therefore, we chose molecule as the level of products inspired by self-assembly. In cases where the design was inspired by secretions by organisms, we chose the smallest biological level at which the role of the secretion was significant to be the categorized biological level of inspiration. Two examples of biomimetic designs inspired by secretions playing role at different biological levels have been provided in Section 3.4.

In order to analyze the effect of visible biological phenomenon on the quantity and quality of biomimetic designs, we formally defined a layman visible scale. Our goal in defining the layman visible scale was to capture some limited set of biological knowledge that a design engineer would have for using bio-inspiration, without actively seeking aid from experts in biology. If the source of bio-inspiration fell on the layman visibility scale, it probably implied that the identification of the bio-inspiration required a lower degree of insight into biology by engineering designers. In such cases, the bio-inspiration could be identified by engineering designers with little or no expert aid. However, this could also imply that the identified bio-inspiration was serendipitous or well known. Thus, we used the layman visible scale as a basis to analyze the novelty of the biomimetic products inspired by serendipitously and obviously discovered biological entities.

For a level to fall on layman visible scale, two conditions were imposed as follows:

1. The phenomenon occurring at the level should be visible to the naked eye without the use of any aiding device.

2. The level should have a surface interaction with the external environment.

Based on the criteria defined for layman visible scale, we analyzed the 'layman visibility' for each level in the biological organizational scale. The levels of molecule, organelle and cell are only visible using microscopes and other magnifying devices and hence, are not layman visible.

The levels of organ and tissue, in general, are visible to the unaided naked eye, but in general cannot be categorized as falling on the layman visible scale as a whole. For example, internal tissues and organs, such as bones and muscles, do not interact with the external environment, that is the atmosphere and function inside the body. By contrast, skin and leaves have direct interaction with 
the external environment and hence satisfy both conditions of layman visibility. However, a closer analysis reveals that about $83 \%$ of the designs inspired by organs and tissues fall on the layman visible scale. Hence, we attributed both levels of tissue and organ as layman visible.

The level of organ system, despite being visible without any aid, do not have surface interactions with the external environment. Thus, they violate one condition of layman visibility and, therefore, can be kept out of the layman visible scale.

Levels of organism and population are generally visible to the naked eye, with the exception of microscopic organisms and population. We analyzed that only $6 \%$ of the biomimetic designs inspired in the categories of organism and population are inspired by microscopic life forms. Therefore, the levels of organism and population were attributed as layman visible.

The ecosystem level is somewhat too large to be visible as a whole to the unaided naked eye, therefore, we chose it to be kept out of the layman visibility scale.

The layman visible scale was finally comprised of four levels of biological organization: tissues, organs, organism, and population.

\subsection{Abstraction Level}

Biomimetics involves mapping of information from biology to the technology using analogical reasoning [2,35]. More precisely, a worthwhile biological phenomenon is carried-over to technology to develop a novel solution for some identified problem in technology [11,35]. The degree of biology to technology carry-over can vary depending upon the requirement and the state of knowledge of the designer [11]. We used the abstraction level to qualitatively define the degree of carry-over from biology to technology for each design. The abstraction level refers to the hierarchical level at which the design was manipulated in technology to achieve the biomimetic solution to the respective problem. The abstraction level has been classified in multiple ways in the literature. Vincent identified six categories of operational fields (analogous to design entities) in an attempt to qualitatively differentiate between the solutions provided by nature and technology [4]. A problem, be it in technology or nature, could be solved by manipulation of one or more of the following fields: substance, structure, space, time, energy and information [4]. The nature of biological phenomenon can also be classified into three categories: form, behavior, and principles [11]. This form of categorization by Mak and Shu is hierarchical in nature, that is, form is a subset of behavior which, in turn, is a subset of principles. Vincent studied the biomimetic products which were ready to be introduced or had been introduced in the market by classifying them into three broad categories: materials, surface structures, and mechanisms [23]. Bio-inspired products have also been classified based on the type of engineering problem solved by using inspiration from biology [24,36].

We used a modified version of Mak and Shu's method for the classification of the abstraction levels. The categories of behavior and principle were used in the original context. In order to elaborate on the variety of mimicked biological phenomenon, we split the category of form in two categories. An inspiration of form mimics the materials, structures, mechanisms or processes in biology [23]. However, the inspiration from biological materials and structures was observed to overlap in some cases where the structure of the biological material serves as the inspiration for the design. Therefore, to avoid ambiguity, we used a collective category of 'Materials and Structures'. A similar ambiguity was observed in case of mechanisms and processes, so we merged both categories into a single category of 'Mechanisms and Processes'. Hence, we qualitatively defined the abstraction level of the biomimetic designs by classifying them into four categories. The four categories form an abstraction hierarchy as shown in Figure 1. 


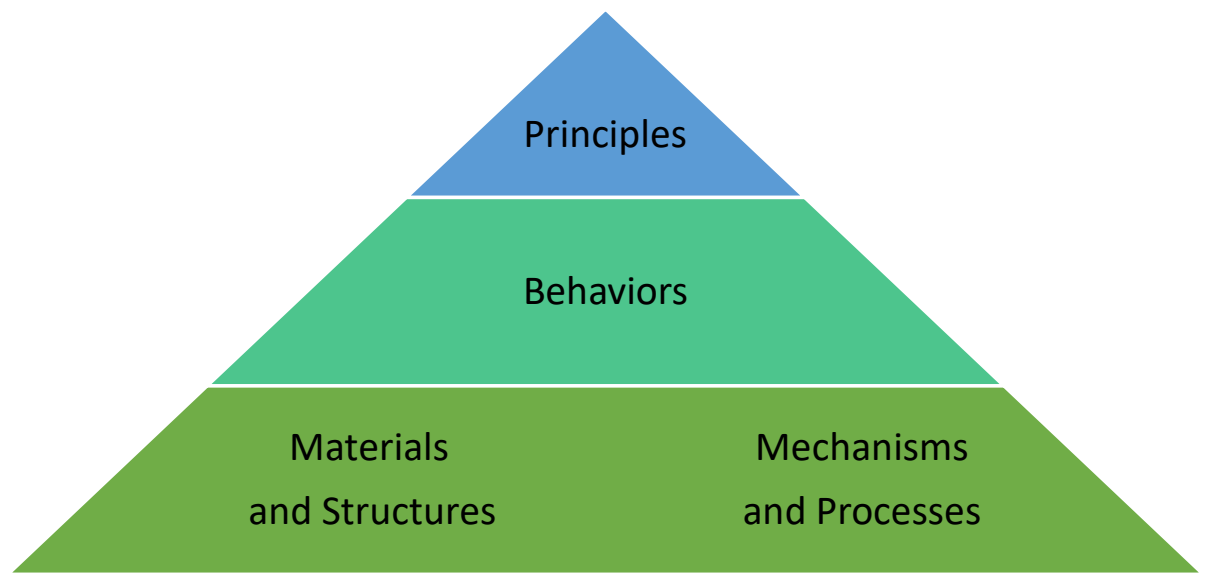

Figure 1. Hierarchy of abstraction levels, adapted from Mak and Shu [11].

\subsection{Novelty}

Using the criteria of novelty for categorization is an assessment of design innovation. Innovation measures of a product are of crucial importance to engineering. Although the term 'innovation' is used equivocally with 'innovativeness', the two terms are disparate [26,37]. Assessing the measure of innovation requires consideration of both technological and market perspectives of a design, while the measure of innovativeness considers only the technological perspective [26]. Innovativeness is most frequently used as a measure of newness of a product, independent of its effect on the market [26]. As the designs/products in the sample space are limited to implementation or higher phase, therefore, we find it convenient to determine the innovation measure by only considering the technological perspective of the design. However, we acknowledge that the market perspective is no less important than the technological perspective while determining the innovation measure. Both innovation and innovativeness are multi-dimensional measures [26,38-40]. At least fifteen constructs have been used to model innovativeness which cumulatively use fifty-one scale items to measure innovativeness [26].

Despite the representation of innovativeness in multiple ways, innovativeness of a product has always been modeled as the degree of discontinuity in marketing and/or technological factors [26,40]. In order to qualitatively define innovation and innovativeness, multiple methods of categorization have been proposed and practiced in the literature. The methods are mostly based on the type and degree of discontinuity a product causes in the market [26,41-43]. Novelty has been identified as an important driver of innovation [44,45]. There exist multiple methods of assessing the novelty of a product, which include qualitative grading of the function satisfied by a product or an idea [46], counting of distinct solution ideas based on prior knowledge $[44,46-48]$ and the citation information of an idea [49].

Sarkar and Chakrabarti identified that most of the methods for assessing novelty focused on identifying whether a design is novel or not, and not on defining the degree of novelty, and proposed a method to determine the qualitative measure of creativity of a product by considering two constructs of creativity as novelty and usefulness [25]. Since creativity plays a crucial role in empowering innovation, it can be deemed as a measure of innovation of a product, where the novelty and usefulness are analogous to innovativeness and market impact [25]. Sarkar and Chakrabarti analyzed the pairwise correlation between novelty, usefulness and creativity, and found that the novelty of a design has a greater impact on creativity than its usefulness [25]. Moreover, the market success of a product depends upon its degree of novelty [38]. Thus, the qualitative measure of novelty was chosen as a measure of innovativeness.

We used Sarkar and Chakrabarti's method to assess the novelty of identified biomimetic designs. The method qualitatively categorizes products into four categories based on their novelty: very high novelty products, high novelty products, medium novelty products, and low novelty products. The novelty of a product is determined by comparing the Function-Behavior-Structure (FBS) model or 
the SAPPhIRE constructs of the product with those of the already existing products. The function of a system/design is a description of what the system does and is at a higher level of abstraction than behavior. The behavior is defined as how the intended function is achieved by the structure of the design [50]. The SAPPhIRE model consists of eight constructs arranged in a hierarchical structure. The mapping of the SAPPhIRE model to the FBS model is shown in Figure 2.

Sarkar and Chakrabarti's method analyzes the difference in the constructs of the new and existing products to determine the relative degree of novelty of the new product. The highest construct at which the new product can be different from the existing product is then identified. The higher the hierarchy of the construct at which the difference lies, the higher the novelty score of the new product [25].

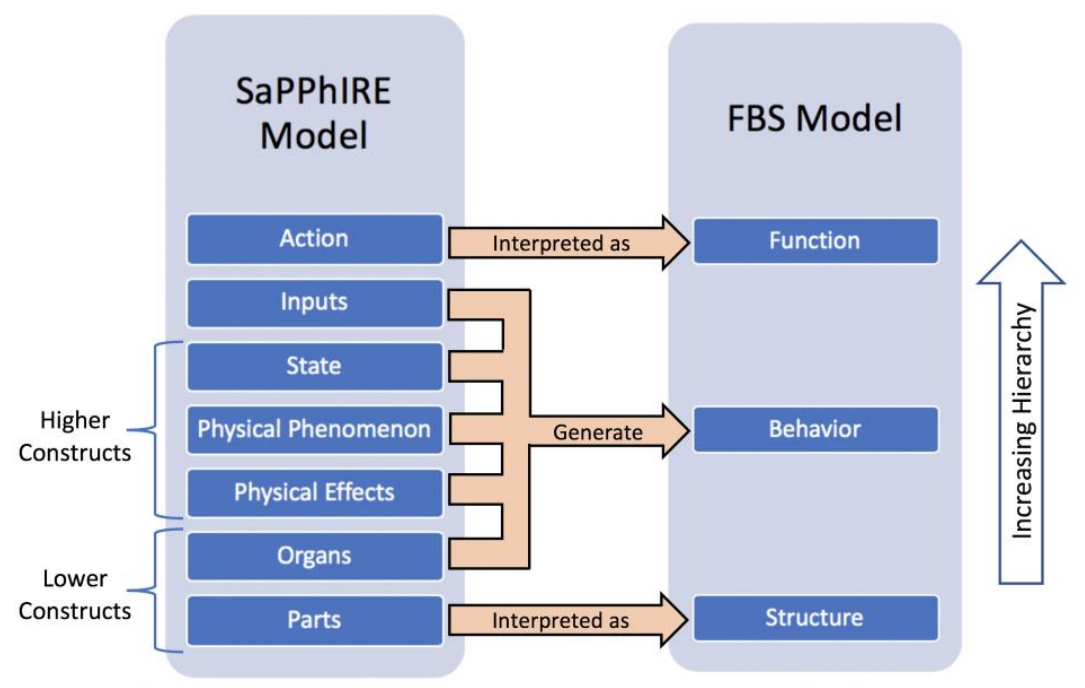

Figure 2. Mapping of SAPPhIRE model to FBS model, adapted from Sarkar and Chakrabarti [25].

Since the qualitative impact of the biomimetic solutions on technology was to be analyzed, we assessed the novelty of the biomimetic products by comparing their FBS and SAPPhIRE constructs to the products existing in current technology and not with other biomimetic designs. We will refer to the technological products relevant to the biomimetic product being analyzed as 'existing products'. We determined the FBS and SAPPhIRE constructs for each biomimetic product. In some cases, the AskNature database provided the difference between the biomimetic product, and the existing products. In other cases, the differences were sought for and found in the literature. The identified differences between the biomimetic product and the new product were then mapped to the SAPPhIRE model. Based on the comparison of the SAPPhIRE constructs, products can be classified as follows [25]:

Very High Novelty Product: If the biomimetic product differs from existing products at function or action levels, the biomimetic product is attributed as a very high novelty product. Very highly novel biomimetic products generally result in the introduction of new functions in existing technology.

High Novelty Product: If the biomimetic product and existing products perform the same function (action) and the biomimetic product differs from existing products in the SAPPhIRE construct of input or state change (interpretation of the input), the biomimetic product is attributed as a high novelty product. High novelty biomimetic products generally result in new approaches to perform functions already performed by existing technology.

Medium Novelty Product: If the biomimetic product and existing products are similar in constructs of action, input and state change, and the new product differs from existing products in terms of physical phenomenon or physical effect, the new product is attributed as medium novelty product. The medium novelty biomimetic products achieve functions already performed by the current technology. However, the approach differs at a lower level of abstraction as compared to high novelty products. 
Low Novelty Product: If the new product is only a structural variant of an existing product the new product is attributed as a low novelty product. In this case, the new and the existing product perform the same function (action) and exhibit similar behavior. The difference lies in the organ or part level. Low novelty biomimetic products achieve functions already performed by the current technology using the same approach but in a more efficient way.

In order to assess the novelty of a design, the design needs to be sufficiently detailed to determine its FBS or SAPPhIRE constructs. However, certain biomimetic designs reviewed indicated only the behavior of the design and not design function. For example, one of the biomimetic designs was the mimicry of the structure of the shark skin which resulted in a reduced drag [51]. Drag reduction is a form of behavior, which ultimately helps in achieving a function which was not explicitly defined in the literature describing the shark skin suit [52]. The essence of bio-inspiration lies in the fact that the inspiration either provides a new application in technology or improves the performance of current technology in an existing application. Therefore, in such cases where only behavioral aspects of the design could be identified, we assumed that the design aided in satisfying a function already existing in the technology. The non-existence of a unique function in some biomimetic design automatically meant that the design was not very highly novel, because the design function was already achievable by current technology. Subsequently, we used the remaining SAPPhIRE constructs to determine whether the novelty of the biomimetic product is high, medium or low. The sequence of steps followed for assessing novelty of the biomimetic products is shown in Figure 3.

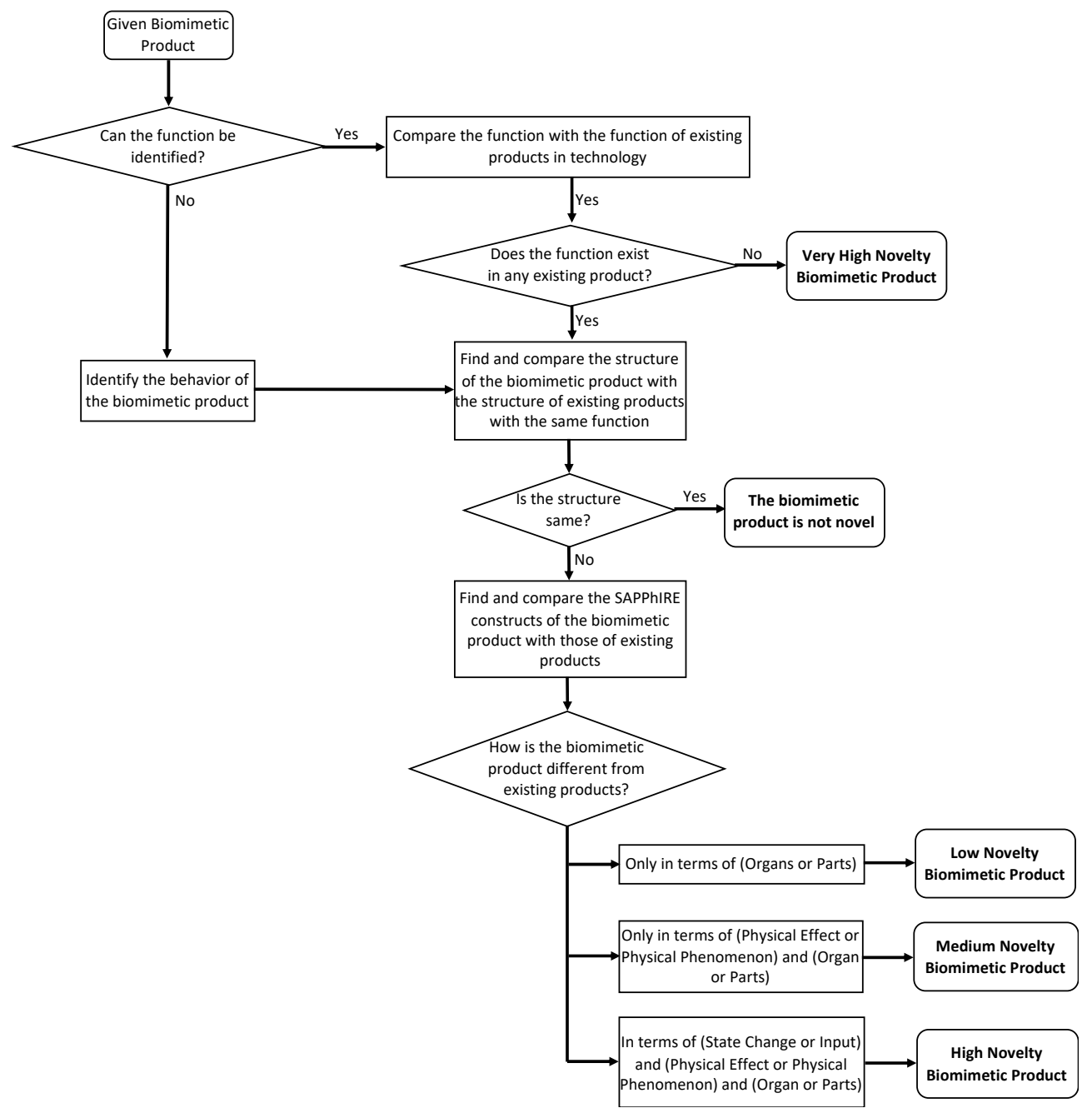

Figure 3. Flowchart for assessing novelty of biomimetic products, adapted from Sarkar and Chakrabarti [25]. 


\subsection{Categorization of Biomimetic Products}

This section provides detailed analysis and classification of biomimetic products according to the criteria described in prior sections. To carefully illustrate the process and provide a roadmap for future biomimetic design analysis, the example classification of seven biomimetic products is presented in some detail in the following sub-sections. The summary of these classifications is shown in Table 1.

Table 1. Full classification of seven biomimetic designs.

\begin{tabular}{cccc}
\hline Product/Application & Biological Organizational Level & Abstraction Level & Novelty \\
\hline Self-Healing Concrete & Tissue & Behavior & Very High \\
Pipeline Leak Sealer & Organ System & Mechanism & High \\
Water Management System & Ecosystem & Principles & High \\
Self-cleaning Paint & Organ & Structure & Medium \\
Surgical Glue & Organism & Material & Medium \\
Vibro-Wind & Organ & Behavior & Medium \\
Anti-fouling Coatings & Organ & Material & Low \\
\hline
\end{tabular}

\subsubsection{Self-Healing Concrete}

Description: Self-healing is a behavior commonly exhibited in biological systems. Inspired by the self-healing behavior observed in biology, researchers have developed a new form of concrete [53]. Structures built using the biomimetic concrete allows concrete structures to be more flexible and hence, avoid fracturing. Even if fracturing does occur, the concrete at the location of the crack absorbs moisture from the surrounding air, grows until the crack, and finally solidifies to its original strength.

Biological Organizational Level: The self-healing behavior can be observed occurring at multiple levels on the biological organizational scale. However, the smallest level at which the self-healing phenomenon has been observed is at the cellular level. Therefore, the biological organizational level for the biomimetic product is cell.

Abstraction Level: The biomimetic product and existing products do not have the same structure, material, or mechanism. Rather, the self-healing behavior of biological systems serves as the inspiration to develop self-healing biomimetic product. Hence, the abstraction level is behavior.

Novelty: The new biomimetic product performs the function of 'self-healing'. Existing products are not capable of performing the same function as the biomimetic product. Therefore, the biomimetic product is a very high novelty product.

\subsubsection{Pipeline Leak Sealer}

Description: Platelet Technology ${ }^{\mathrm{TM}}$ was discovered by Dr. Ian McEwan when he cut his finger on a sheet of paper. It is an innovative way of sealing and locating leaks in a single integrated process, based on how human blood carries platelets to the surface of a wound to stop the bleeding [54]. Whereas conventional leak sealing techniques require direct access to the leak, Platelet technology can be implemented remotely, mimicking the healing process in the human body to work with the pressure in the pipeline and seal from the inside.

Biological Organizational Level: The product is inspired by the human circulatory system. The platelets are unable to perform the function unless they are carried by the blood to the leaking area. Therefore, the biological organizational level is organ system.

Abstraction Level: The biological mechanism of internal leak sealing has been mimicked to perform the same function in technology. Therefore, the abstraction level is mechanisms and processes.

Novelty: The function of the biomimetic product is detection and healing of defects in pipelines. Current technology is capable of performing this function, although not autonomously. Further, the difference in the biomimetic and existing products lies in the method of a physical phenomenon of detection and healing. Therefore, the biomimetic product is categorized as a high novelty product. 


\subsubsection{Water Management System}

Description: In an ecosystem, vegetation provides a damping mechanism that slows and redirects the surface water run-off, allowing it to soak into the soil. This damping phenomenon is distributed throughout the whole ecosystem so that no matter where rain falls, the surface water movement is slowed, and absorbed or stored in rivers and lakes to be used by the ecosystem's inhabitants. This.water management technique is serving as inspiration for designing cities that better manage the stormwater runoff [29]. The new biomimetic design prevents overflows from sewer systems during storms, results in lesser soil erosion, and prevents damage by reducing the speed of stormwater runoff.

Biological Organizational Level: The surface water management technique has been observed in natural ecosystems. Thus, the biological organizational level is ecosystem.

Abstraction Level: The product does not mimic the form or the behavior of a natural ecosystem. Rather the natural principles of water management serve as the inspiration for designing cities. Therefore, the abstraction level is principles.

Novelty: The biomimetic product (city) manages stormwater runoff more effectively than the existing products. Therefore, the existing products are capable of performing the function performed by the new biomimetic product. The input to the product is stormwater, which is the same for the new and existing products. However, the difference exists in the way the input (water) is handled, that is, the SAPPhIRE construct of state change. Therefore, the biomimetic product is a high novelty product.

\subsubsection{Self-Cleaning Paint}

Description: Many plant surfaces remain dirt-free, simply by how their complex surface topography interacts with the physics of water molecules [55]. This self-cleaning ability is a result of high degree of hydrophobicity of the microstructure. Lotusan exterior coating, inspired by self-cleaning ability of lotus leaves, mimics this structural strategy to regain its cleanliness automatically after the mere rinse of a rain shower. Existing self-cleaning surfaces achieve hydrophobicity due to their chemical nature, this limits the material type that can be used.

Biological Organizational Level: The lowest biological entity at which the phenomenon is seen is the leaves of lotus plant, which are regarded as the organs of the plant. Thus, the biological organizational level is organ.

Abstraction Level: The microstructure of the lotus leaf surface has been mimicked to exhibit superhydrophobic behavior and thus perform the same function in the engineered domain as it does in the biological domain. Therefore, the abstraction level is mechanisms and processes.

Novelty: Super-hydrophobic materials do exist in current technology; therefore, the function can, in principle, be performed by existing products. But existing products achieve superhydrophobicity by the virtue of their chemical nature, while the biomimetic product achieves superhydrophobicity by the virtue of its surface microstructure. Therefore, the physical phenomenon on which the biomimetic product is based is different from the existing products. Therefore, the new biomimetic product is a medium novelty product.

\subsubsection{Surgical Glue}

Description: During surgery, it may be difficult to apply stitches to internal wounds without damaging the tissue or to use surgical glues without the glues being washed away. Gecko Biomedical has designed a bioinspired light-activated tissue adhesive that works as a surgical sealant [56]. The properties of this adhesive gel are inspired by sticky and quick-setting secretions produced by sandcastle worms. The worms use the secretions to bind building materials (such as sand) together to build their home. 
Biological Organizational Level: The product is inspired by the properties of secretions of sandcastle worms. Since the secretion plays a role in the protection of the sandcastle worm, the biological organizational level is organism.

Abstraction Level: The material secreted by the sandcastle worm is mimicked to develop a product that performs better than existing surgical glues. Hence, the abstraction level is structures and materials.

Novelty: The new biomimetic product has been developed to enhance the properties of existing surgical glues. Therefore, the function performed by the new product can be performed by existing products. The material properties of the new biomimetic product are different from that of existing products performing the same function. The highest level in the SAPPhIRE model at which the new product differs from existing products exists in the way the glue interacts with its environment, that is the construct of physical phenomenon. Therefore, the product is a medium novelty product.

\subsubsection{Vibro-Wind}

Description: Current wind harvesting methods use rotary turbines to generate electricity. However, the existing methods have multiple shortcomings which include noise generation, danger to birds, and difficultly of implementation in urban environments. Inspired by the oscillatory movement of leaves in the wind, researchers at Cornell University have developed a mechanism which utilizes leaf-like vibratory motion to harvest energy [29]. The mechanism is capable of functioning in variable wind speeds, and generates little noise and vibration.

Biological Organizational Level: The energy harvesting mechanism has been inspired by the movement of leaves. Since leaves are regarded as organs of a plant, the biological organizational level is organ.

Abstraction Level: The vibratory motion of leaves is mimicked to develop a mechanism for harvesting wind energy. In nature, the motion is not used to harness energy. Hence, the abstraction level is behavior.

Novelty: The new biomimetic product has been developed to counter the limitations in existing wind harvesting methods. Therefore, the function performed by the biomimetic product can be performed by the existing products. The input to the product is wind, which is the same for the new and existing products. The state change is from wind energy to mechanical energy, which is also the same as existing products. However, the physical effect of wind on the biomimetic product is vibration/oscillation, while it is rotation in existing products. Therefore, the highest level in the SAPPhIRE model at which the new product differs from existing products exists at the SAPPhIRE construct of physical effect. Hence, the product is categorized as a medium novelty product.

\subsubsection{Anti-Fouling Coatings}

Description: Some organisms like fish have a coating consisting of bacteria and other substances on their skin. This coating repels undesirable organisms from attaching themselves to the skin of fish, and slowing the fish down. The repulsive coating contains only solids and is devoid of any solvents. Eco-Clad ${ }^{\circledR}$ paint is inspired by these biological coatings and eliminates the use of VOCs (volatile organic compounds) and other toxicity issues of existing biocides [29]. Eco-Clad paint provides a more environment-friendly way to prevent biological fouling of boats.

Biological Organizational Level: The product is inspired by the properties of fish secretions. Since the secretion plays a role in protecting fish skin from biological fouling, the biological organizational level is tissue.

Abstraction Level: The material secreted by fish is mimicked to develop a product which is more bio-friendly than existing anti-fouling paints. Hence, the abstraction level is structures and materials. 
Novelty: The new biomimetic product has been developed to enhance the characteristics of existing anti-fouling paints, therefore the function performed by the product can be performed by the existing products. The interaction of the product with its environment is the same, that is, repulsion. The material properties (SAPPhIRE construct of structure) are different from existing product. Therefore, the product is categorized as a low novelty product.

The examples illustrated above show the systematic approach used to recognize, understand, and categorize each of the biomimetic products in the sample set. To assure reliability of the categorization performed in this study, we measured the inter-rater agreements for the categorization of biomimetic products performed in this study. The biological organizational scale was observed to be free of subjectivity, therefore we measured the inter-rater agreements only for the categories of abstraction level and novelty. Prior to this work, Srinivasan and Chakrabarti assess the subjectivity in categorizing design outcomes into one of the SAPPhIRE constructs and found acceptable agreement score [57]. The biomimetic products in this study were rated by two raters. Both the raters belonged to different geographical, educational and cultural backgrounds, but had prior knowledge about biomimetics and bio-inspired engineering design. One of the raters classified all of the products in the sample, while the other classified twenty products selected at random from the sample. The abstraction level scale formed a nominal scale of categorization; therefore, we used Cohen's Kappa coefficient for assessing the inter-rater agreement $[58,59]$. The Cohen's Kappa coefficient was found to be 0.7989 for the scale of abstraction level, which suggested substantial agreement [60]. The novelty rating formed an ordinal scale of categorization; therefore, we used weighted Cohen's Kappa coefficient with linear weights was used for assessing the inter-rater agreement [59,60]. The weighted Cohen's Kappa coefficient was found to be 0.8118 for the novelty, which suggested almost perfect agreement [60].

\section{Results and Discussion}

In order to predict the pattern in existing biomimetic designs, we analyzed their distribution among the three categories in five possible combinations. The first distribution involved arrangement of designs according to the level of biological organization and the abstraction level. Our intent was to identify the prominent biological levels serving as inspiration for each abstraction level. The other four combinations involved the spread of novelty across the biological levels for each type of abstraction level. Our intent was to identify the biological levels which tend to inspire biomimetic designs with high and very high novelty score. The term 'good novelty' is used to refer to designs with very high and high novelty.

\subsection{Level-Nature Distribution}

The distribution of the abstraction levels across the levels of biological organizational scale is shown in Figure 4. On the biological organizational scale, the organism level accounts for the highest number $(36 \%)$ of the total generated biomimetic designs. The layman visibility scale accounts for $73 \%$ of total analyzed biomimetic designs. As we go higher in the hierarchy of abstraction level, the number of biomimetic designs in the particular abstraction level tends to decrease. 


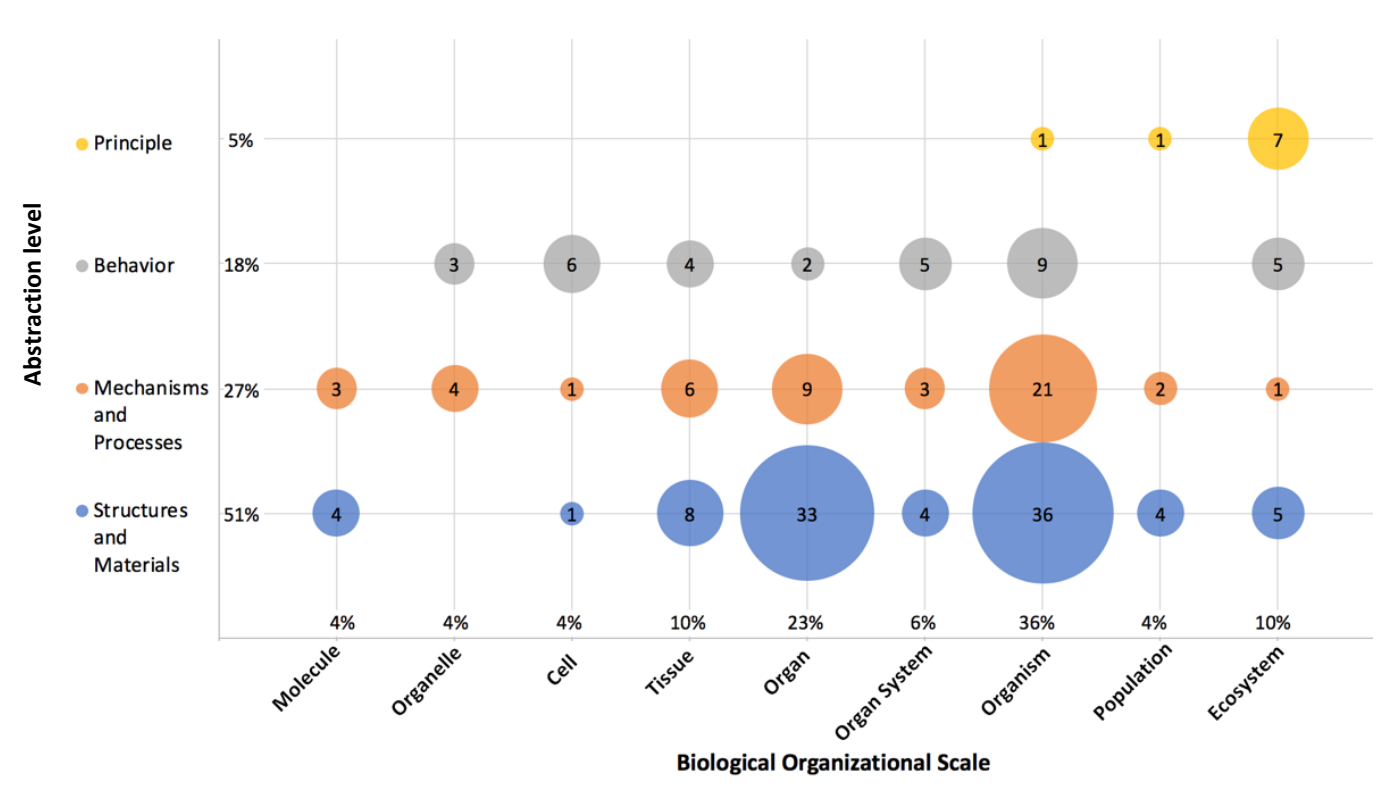

Figure 4. Level-Nature Distribution of Biomimetic Designs $(n=188)$.

\subsection{Biomimicry of Structures and Materials}

The novelty distribution according to the level of biological organization for the inspiration domain of structures and materials is shown in Figure 5. The organism level accounts for the highest number $(38 \%)$ of all the designs mimicking biological structures and materials. $85 \%$ of the analyzed biomimetic structures and materials are inspired by the organizational levels falling on the layman visible scale. We observed the biological organizational levels of organelle and cell to be the lowest contributors to the development of biomimetic structures and materials. However, very few $(6 \%)$ of the biomimetic structures and materials were found to a good novelty score. This is because most of the designs mimicking structures and materials only describe the behavior of the biomimetic design and not its function. Consequently, we can say that the biomimetic designs analyzed in the study mostly focus on improving the functionality of the existing technological designs and rarely introduce new functions. Moreover, we observed that a substantial number $(87 \%)$ of the low novelty biomimetic products fall on the layman visible scale.

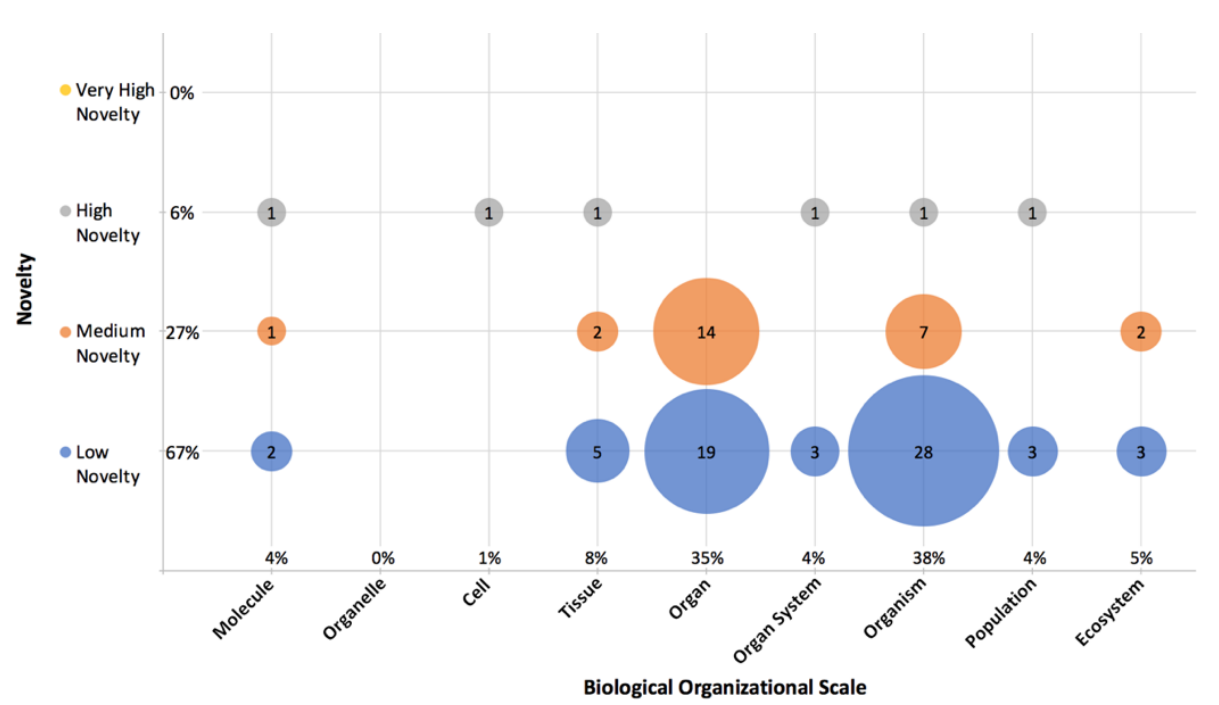

Figure 5. Novelty Distribution of Biomimetic Structures and Materials $(n=95)$. 


\subsection{Biomimicry of Mechanisms and Processes}

The distribution of the novelty of biomimetic mechanisms and processes according to the level of biological organizational scale is shown in Figure 6. The mimicry of biological mechanisms and processes spans across all the levels on the biological scale. The organism level accounts for highest number $(40 \%)$ of all biomimetic mechanisms and processes. $76 \%$ of the analyzed biomimetic structures and materials are inspired by the organizational levels falling on the layman visible scale. $34 \%$ of the designs mimicking biological mechanisms and processes have a good novelty score. The designs with the highest novelty score were found at the tissue levels. We observed biological organizational levels of cell and ecosystem to be the lowest contributors to bio-inspired mechanisms and processes.

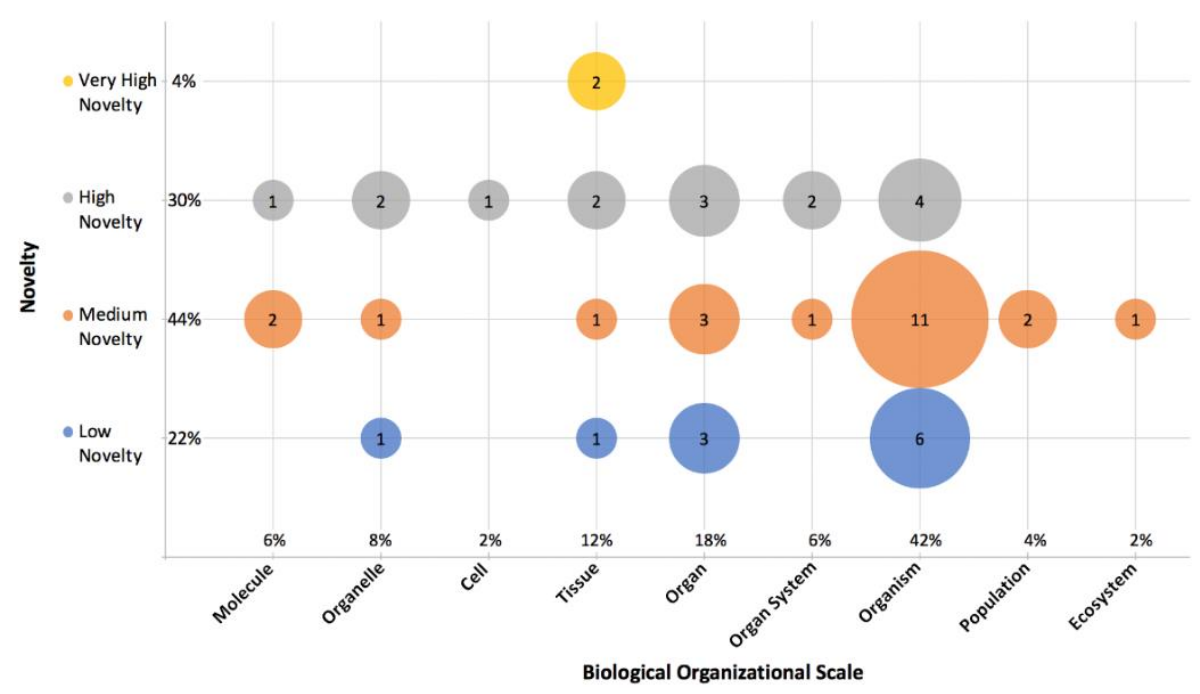

Figure 6. Novelty Distribution of Biomimetic Mechanisms and Processes $(n=50)$.

\subsection{Biomimicry of Behavior}

The distribution of the novelty of the designs mimicking biological behavior according to the level of biological organization is shown in Figure 7. The organism level inspires the highest number (26\%) of designs mimicking biological behavior. In contrast to the biomimicry of form, $76 \%$ of the designs mimicking biological behavior have a good novelty score. The designs with the highest novelty score were found at the organelle, tissue, organ system and the organism level.

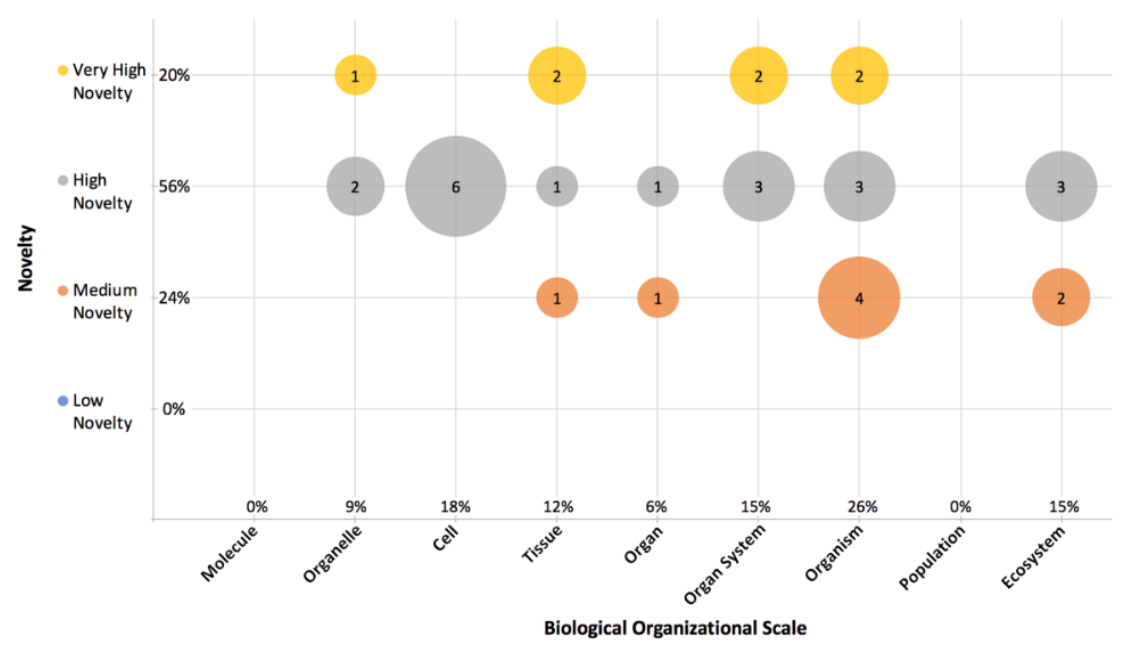

Figure 7. Novelty Distribution of Biomimetic Behaviors $(n=34)$. 


\subsection{Biomimicry of Principles}

The distribution of the novelty of designs mimicking biological principles according to the level of biological organization is shown in Figure 8. Due to comparatively lesser number of biomimetic designs inspired by biological principles in the analyzed set, the statistical significance of cross-categorical study is comparatively lesser than other abstraction levels. However, we observed that all the designs mimicking biological principles are inspired by biological entities at organism or higher organizational levels. The Ecosystem level serves as the most prominent biological level in the inspiration of biological principles. Moreover, we found a substantial proportion of the total number of biomimetic products inspired by biological principles to have a good novelty score. The distribution shows that the principles governing biological phenomena occurring at biological levels lower than the organism level might not have been effectively used in development of technology.

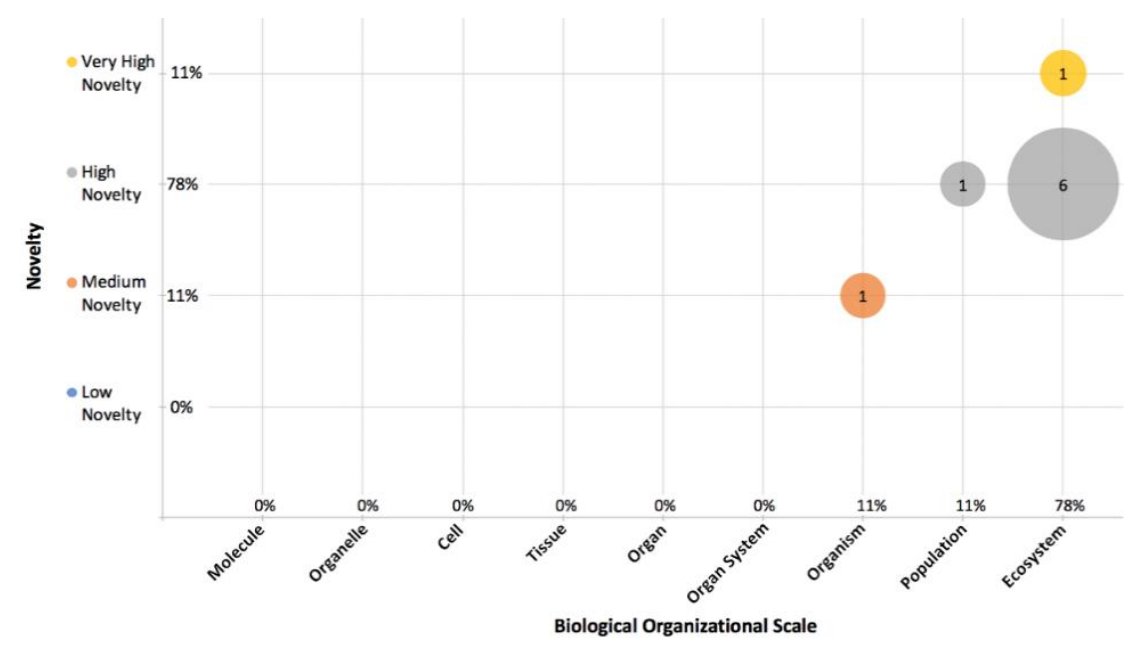

Figure 8. Novelty Distribution of Biomimetic Principles $(n=9)$.

\section{Conclusions and Future Work}

In this work, we presented an approach for determining patterns in biomimetic designs by categorizing biomimetic products according to the biological organizational level, the abstraction level, and novelty. The sample set used in this study indicates a pattern in novelty of existing biomimetic products. Utilizing the pattern observed in the novelty of existing biomimetic products, practitioners of biomimetics can identify the prominent biological organization levels, and determine the degree of biology to technology carry-over required for generating novel biomimetic solutions to engineering problems. Moreover, researchers can utilize the suggested method to identify the less explored biological levels that may carry potential bio-inspirations.

Firstly, we defined a layman scale to account for describing inspiration from the visible and well-known biological entities. The study indicates that the layman visible scale, specifically, the organism level is the most preferred level for the exploration of biomimetic structures, materials, mechanisms and behavior. This suggests that most of the existing biomimetic designs required lower degrees of insight into biology by engineering designers, and/or are inspired by serendipitous and well-known analogies.

The study indicates structures and materials are the most prominent area of biomimetic design. Most of the biomimetic materials and structures were found to be inspired by the biological organizational levels falling on the layman visible scale. However, the novel biomimetic materials and structures were also found to occur at non-layman visible scale levels of molecule, cell and organ system, which are comparatively less explored by engineering designers. In addition, we could not find highly novel biomimetic structures and materials in the sample set. This may indicate that biomimetics has seldomly resulted in development of novel functions in the field of structures and materials. 
Almost all of the biological scale was found to be utilized for the development of mechanisms and processes. However, most biomimetic mechanisms and processes are inspired by biological organizational levels falling on the layman visible scale. As the most novel biomimetic designs result in addition of new functions to existing technology and provide new approaches to performing existing functions, the study indicates that the biological organizational levels are lower than at the organism level, and particularly tissue level, to be the source of inspiration for novel mechanisms and processes.

In general, the mimicry of biological behaviors and principles was found to result in higher novelty products. Thus, more focus needs to be placed in inculcating biological behaviors and principles in engineering designs, rather than just mimicking the form of biological entities. Biological behaviors occurring at molecule and population level were found to be less explored for generation of biomimetic designs. Moreover, the study indicates that nature's pool of design principles remains highly underutilized. The existing biomimetic products based on biological principles utilize the biological principles from the population and ecosystem levels. The study suggests that biomimetic researchers should explore the biological principles governing the phenomena occurring at other levels to develop products with good novelty.

The study presents the application of cross-categorical analysis to the field of bio-inspired design, and biomimetics without being specific to the domain of application. The method can be applied by biomimetic practitioners, while restricting the designs in the sample set to their relevant field of interest, to identify prominent biological organizational levels, and abstraction levels which have resulted in development of novel biomimetic products in the past. Since the measure of novelty, as used in the study, provides the qualitative comparison of the solution provided by the biomimetic design with the conventional form of designs, the identified prominent categories can possibly inspire solutions to design problems beyond the scope of present technology.

The methods of identifying biomimetic designs in the current study are restricted to manual and database search. We intend to collect a more extensive biomimetic sample set that can be used for training autonomous classification and bio-inspiration search tools. Additionally, we intend to identify and normalize the approaches used in development of biomimetic products identified as very highly and highly novel. However, our study considers only the novelty of the designs and not the full measure of innovation. In order to include the market impact of biomimetic products, the full dimension of creativity, that is, both novelty and usefulness, could be analyzed to more accurately predict the success of biomimetic products in the market.

Author Contributions: The contributions of the respective authors are as follows: D.B. performed research and analysis; D.A.M. supervised the analysis and research, and edited and revised the manuscript.

Funding: This research received no external funding.

Acknowledgments: The authors would like to thank Elissa Morris, Department of Mechanical Engineering, Texas A\&M University for her role as inter-rater in this study.

Conflicts of Interest: The authors declare no conflict of interest.

\section{References}

1. Salgueiredo, C.F.; Hatchuel, A. Beyond analogy: A model of bioinspiration for creative design. AI EDAM 2016, 30, 159-170. [CrossRef]

2. Fayemi, P.E.; Wanieck, K.; Zollfrank, C.; Maranzana, N.; Aoussat, A. Biomimetics: Process, tools and practice. Bioinspir. Biomim. 2017, 12, 011002. [CrossRef] [PubMed]

3. Wanieck, K.; Fayemi, P.E.; Jacobs, S. Biomimetics and its tools. Biomim. Its Tools 2017, 6, 53-66. [CrossRef]

4. Vincent, J.F.; Bogatyreva, O.A.; Bogatyrev, N.R.; Bowyer, A.; Pahl, A.-K. Biomimetics: Its practice and theory. J. R. Soc. Interface 2006, 3, 471-482. [CrossRef] [PubMed]

5. Fayemi, P.E.; Maranzana, N.; Aoussat, A.; Bersano, G. Bio-inspired design characterisation and its links with problem solving tools. In Proceedings of the DS 77: Proceedings of the DESIGN 2014 13th International Design Conference, Croatia, Dubrovnik, 19-22 May 2014. 
6. Helms, M.; Vattam, S.S.; Goel, A.K. Biologically inspired design: Process and products. Des. Stud. 2009, 30, 606-622. [CrossRef]

7. Weidner, B.V.; Nagel, J.; Weber, H.J. Facilitation method for the translation of biological systems to technical design solutions. Int. J. Des. Creat. Innov. 2018, 1-24. [CrossRef]

8. Speck, T.; Speck, O.; Beheshti, N.; McIntosh, A.C. Process sequences in biomimetic research. Des. Nat. IV 2008, 114, 3-11.

9. Badarnah, L.; Kadri, U. A methodology for the generation of biomimetic design concepts. Arch. Sci. Rev. 2015, 58, 120-133. [CrossRef]

10. Shu, L.; Ueda, K.; Chiu, I.; Cheong, H. Biologically inspired design. CIRP Ann. Manuf. Technol. 2011, 60, 673-693. [CrossRef]

11. Mak, T.; Shu, L. Abstraction of biological analogies for design. CIRP Ann. Manuf. Technol. 2004, 53, 117-120. [CrossRef]

12. Jacobs, S.R.; Nichol, E.C.; Helms, M.E. "Where Are We Now and Where Are We Going?" The BioM Innovation Database. J. Mech. Des. 2014, 136, 111101. [CrossRef]

13. Goel, A.K. Design, analogy, and creativity. IEEE Expert 1997, 12, 62-70. [CrossRef]

14. Yen, J.; Weissburg, M. Perspectives on biologically inspired design: Introduction to the collected contributions. Bioinspir. Biomim. 2007. [CrossRef]

15. Vattam, S.S.; Helms, M.E.; Goel, A.K. Nature of creative analogies in biologically inspired innovative design. In Proceedings of the Seventh ACM Conference on Creativity and Cognition, Berkeley, CA, USA, 26-30 October 2009; pp. 255-264.

16. Gebeshuber, I.C.; Drack, M. An attempt to reveal synergies between biology and mechanical engineering. Proc. Inst. Mech. Eng. Part C J. Mech. Eng. Sci. 2008, 222, 1281-1287. [CrossRef]

17. Baumeister, D. Biomimicry Resource Handbook: A Seed Bank of Best Practices; Biomimicry 3.8: Missoula, MT, USA, 2014.

18. Paris, O.; Burgert, I.; Fratzl, P. Biomimetics and biotemplating of natural materials. Mrs Bull. 2010, 35, 219-225. [CrossRef]

19. Hesselberg, T. Biomimetics and the case of the remarkable ragworms. Naturwissenschaften 2007, $94,613$. [CrossRef]

20. Nagel, J.K.; Nagel, R.L.; Stone, R.B.; McAdams, D.A. Function-based, biologically inspired concept generation. AI EDAM 2010, 24, 521-535. [CrossRef]

21. Liu, K.; Jiang, L. Multifunctional integration: From biological to bio-inspired materials. ACS Nano 2011, 5, 6786-6790. [CrossRef]

22. Lenau, T.A.; Metze, A.L.; Hesselberg, T. Paradigms for biologically inspired design. In Bioinspiration, Biomimetics, and Bioreplication VIII; International Society for Optics and Photonics: Bellingham, WA, USA, 2018; p. 1059302.

23. Vincent, J.F. Biomimetics-A review. Proc. Inst. Mech. Eng. Part H J. Eng. Med. 2009, 223, 919-939. [CrossRef]

24. Domke, M.-L.; Farzaneh, H.H. Research in bio-inspired design-What is its current focus? In Proceedings of the DS 89: Proceedings of the Fifth International Conference on Design Creativity (ICDC 2018), University of Bath, Bath, UK, 31 January-2 February 2018; pp. 314-321.

25. Sarkar, P.; Chakrabarti, A. Assessing design creativity. Des. Stud. 2011, 32, 348-383. [CrossRef]

26. Garcia, R.; Calantone, R. A critical look at technological innovation typology and innovativeness terminology: A literature review. J. Prod. Innov. Manag. 2002, 19, 110-132. [CrossRef]

27. Shah, J.J.; Smith, S.M.; Vargas-Hernandez, N. Metrics for measuring ideation effectiveness. Des. Stud. 2003, 24, 111-134. [CrossRef]

28. ISO. ISO 18458: 2015: Biomimetics-Terminology, Concepts and Methodology; Beuth Verlag: Berlin, Germany, 2015.

29. Deldin, J.M.; Schuknecht, M. The AskNature Database. Available online: http:/ /asknature.org (accessed on 17 September 2018).

30. Liu, Y.; Wang, X.; Fei, B.; Hu, H.; Lai, C.; Xin, J.H. Bioinspired, stimuli-responsive, multifunctional superhydrophobic surface with directional wetting, adhesion, and transport of tater. Adv. Funct. Mater. 2015, 25, 5047-5056. [CrossRef] 
31. Vakili, V.; Shu, L.H. Towards biomimetic concept generation. In Proceedings of the ASME Design Engineering Technical Conference on Design Theory and Methodology, Pittsburgh, PA, USA, 9-12 September 2001; Volume 4, pp. 327-335.

32. Hacco, E.; Shu, L.H. Biomimetic concept generation applied to design for remanufacture. In Proceedings of the ASME 2002 International Design Engineering Technical Conferences and Computers and Information in Engineering Conference, Montreal, QC, Canada, 29 September-2 October 2002; pp. 239-246.

33. Shu, L.; Stone Robert, B.; McAdams Daniel, A.; Greer James, L. Integrating function-based and biomimetic design for automatic concept generation. In Proceedings of the International Conference on Engineering Design (ICED'07), Paris, France, 28-31 August 2007.

34. Whitesides, G.M.; Grzybowski, B. Self-assembly at all scales. Science 2002, 295, 2418-2421. [CrossRef]

35. Shu, L. Biomimetic Design to Enable Sustainable Product Development. In Proceedings of the Global Conference on Sustainable Product Development and Life Cycle Engineering, Berlin, Germany, 29 September-1 October 2004.

36. Murray, K.R. Classification of Biological Phenomena to Aid in Search and Retrieval for Biomimicry; Clemson University: Clemson, SC, USA, 2013.

37. Verhees, F.J.; Meulenberg, M.T. Market orientation, innovativeness, product innovation, and performance in small firms. J. Small Bus. Manag. 2004, 42, 134-154. [CrossRef]

38. Saunders, M.N.; Seepersad, C.C.; Hölttä-Otto, K. The characteristics of innovative, mechanical products. J. Mech. Des. 2011, 133, 021009. [CrossRef]

39. Cooper, R.G. The dimensions of industrial new product success and failure. J. Mark. 1979, 93-103. [CrossRef]

40. Green, S.G.; Gavin, M.B.; Aiman-Smith, L. Assessing a multidimensional measure of radical technological innovation. IEEE Trans. Eng. Manag. 1995, 42, 203-214. [CrossRef]

41. Coccia, M. A New Taxonomy of Technologies; Working Paper CocciaLab; Arizona State University (USA): Tempe, AZ, USA, 2017.

42. Coccia, M. Classifications of Innovations: Survey and Future Directions. Working Paper. CERIS-CNR: Moncalieri (Torino), Italy, August 2006; Anno 8.

43. Abernathy, W.J.; Clark, K.B. Innovation: Mapping the winds of creative destruction. Res. Policy 1985, 14, 3-22. [CrossRef]

44. Verhoeven, D.; Bakker, J.; Veugelers, R. Measuring technological novelty with patent-based indicators. Res. Policy 2016, 45, 707-723. [CrossRef]

45. Arthur, W.B. The structure of invention. Res. Policy 2007, 36, 274-287. [CrossRef]

46. Shah, J.J.; Kulkarni, S.V.; Vargas-Hernandez, N. Evaluation of idea generation methods for conceptual design: Effectiveness metrics and design of experiments. J. Mech. Des. 2000, 122, 377-384. [CrossRef]

47. Saunders, R. Curious Design Agents and Artificial Creativity-A Synthetic Approach to the Study of Creative Behaviour. Ph.D. Thesis, University Sydney, Camperdown, NSW, Australia, 2002.

48. Redelinghuys, C. Proposed criteria for the detection of invention in engineering design. J. Eng. Des. 2000, 11, 265-282. [CrossRef]

49. Park, H.W.; Suh, S.H. Scientific and technological knowledge flow and technological innovation: Quantitative approach using patent citation. Asian J. Technol. Innov. 2013, 21, 153-169. [CrossRef]

50. Chakrabarti, A.; Sarkar, P.; Leelavathamma, B.; Nataraju, B. A functional representation for aiding biomimetic and artificial inspiration of new ideas. AI EDAM 2005, 19, 113-132. [CrossRef]

51. Wen, L.; Weaver, J.C.; Lauder, G.V. Biomimetic shark skin: Design, fabrication and hydrodynamic function. J. Exp. Boil. 2014, 217, 1656-1666. [CrossRef]

52. Dean, B.; Bhushan, B. Shark-skin surfaces for fluid-drag reduction in turbulent flow: A review. Philos. Trans. R. Soc. Lond. A Math. Phys. Eng. Sci. 2010, 368, 4775-4806. [CrossRef]

53. Li, V.C.; Yang, E.H. Engineered self healing cementitious composites. U.S. Patent 7,572,501, 11 August 2009.

54. Evans, K.D.; Ryan, N.J.; McEwan, I.K. Leak location \& sealing using platelet technologyTM. In Proceedings of the OCEANS 2007-Europe, Aberdeen, UK, 18-21 June 2007; pp. 1-4.

55. Spaeth, M.; Barthlott, W. Lotus-Effect ${ }^{\circledR}$ : Biomimetic super-hydrophobic surfaces and their application. In Advances in Science and Technology; Trans Tech Publications: Stafa-Zurich, Switzerland; pp. 38-46.

56. Lang, N.; Pereira, M.J.; Lee, Y.; Friehs, I.; Vasilyev, N.V.; Feins, E.N.; Ablasser, K.; O'cearbhaill, E.D.; Xu, C.; Fabozzo, A. A blood-resistant surgical glue for minimally invasive repair of vessels and heart defects. Sci. Transl. Med. 2014, 6, 218ra216. [CrossRef] 
57. Srinivasan, V.; Chakrabarti, A. Investigating novelty-outcome relationships in engineering design. AI EDAM 2010, 24, 161-178. [CrossRef]

58. Clark-Carter, D. Doing Quantitative Psychological Research: From Design to Report; Psychology Press/Erlbaum (UK) Taylor \& Francis: Abingdon, UK, 1997.

59. Banerjee, M.; Capozzoli, M.; McSweeney, L.; Sinha, D. Beyond kappa: A review of interrater agreement measures. Can. J. Stat. 1999, 27, 3-23. [CrossRef]

60. Viera, A.J.; Garrett, J.M. Understanding interobserver agreement: The kappa statistic. Fam. Med. 2005, 37, 360-363. [PubMed]

(C) 2018 by the authors. Licensee MDPI, Basel, Switzerland. This article is an open access article distributed under the terms and conditions of the Creative Commons Attribution (CC BY) license (http://creativecommons.org/licenses/by/4.0/). 